\title{
Psychiatric emergency services and non-acute psychiatric services utilization during COVID-19 pandemic
}

\author{
Dusan Kolar ${ }^{1}$ (D)
}

Received: 29 June 2020 / Accepted: 31 July 2020 / Published online: 8 August 2020

c) Springer-Verlag GmbH Germany, part of Springer Nature 2020

A retrospective study on emergency service utilization during COVID-19 pandemic conducted by Hoyer and colleagues from Germany and paper titled "Decreased utilization of mental health emergency service during the COVID19 pandemic" published in June 2020, presents a very true reflection on mental health during COVID-19 pandemic. Contrary to the common belief and expectations of mental health services being swamped by patients with psychiatric problems during Covid-19, authors clearly demonstrated a significant decline in emergency mental health services utilization and they elaborated well possible reasons for this finding. The very similar reports come from North America. Goldenberg and Parwani in their recently published paper concluded that the rapid establishment of telepsychiatry preserved or even expanded access to treatment and thereby, among other reasons, the emergency visits declined [1].

However, it is important to acknowledge that service utilization has declined in non-acute mental health services as well. In the mood disorders specialized service at Queen's University in Canada, there is a significant decline in the number of elective admissions of patients with severe and treatment-resistant mood disorders. Bed capacity at mood disorders inpatient service is limited to 14 beds and waiting list has been always lengthy. However, during the COVID19 pandemic only 6-7 beds have been occupied. Referrals for this inpatient unit are coming from six outpatient psychiatrists at the mood disorders outpatient clinic, from the acute care setting (transfers of patients with treatmentresistant mood disorders) and from academic psychiatrists working in the community settings. Despite all these referrals sources, the number of elective admissions significantly declined. Suspension of the leaves of absence for voluntary

Dusan Kolar

kolard@providencecare.ca

1 Mood Disorders Research and Treatment Service, Department of Psychiatry, Queen's University, Kingston, ON, Canada patients, no visitor policy and the lack of group therapies at the ward demotivated some patients to accept elective voluntary admission, but overall there has been evidence of the decline in the number of referrals. Some other hospitals in North America tighten admission criteria, particularly for voluntary admission for substance use disorder [2] which was not the case in our hospital.

It is also worth noting that most of the patients with severe and refractory mood disorders and comorbid anxiety disorders followed in the mood disorders specialized clinic remained stable and well served remotely via telemedicine instead of in-person visits over the last 3-4 months of COVID-19 pandemic. Many patients repeatedly emphasized that social distancing measures and isolation have a positive impact on them as all other people in the community are in the same position which helps with the burden of social isolation due to mental illness. Some patients on a higher functional level reported that the "stay at home" order created a sense of holiday. Patients are well supported by their family members who stay at home during the COVID-19 lockdown and this experience of togetherness obviously was beneficial for patients with mood disorders. Finally, many people are working from home during the COVID-19 pandemic. Working from home is beneficial for some patients with mood and anxiety disorders as they are not exposed to social stress at their work place. As a result of all these factors, the number of patients in the outpatient clinics requesting urgent or earlier appointments has also declined. Telemedicine appears to be so far a good substitute of in-person clinic appointments for follow-up patients. This statement does not apply to new consultations as there are a number of diagnostic issues, therapeutic alliance issues, and potential ethical and medicolegal issues associated with telemedicine [3].

The research evidence on acute psychiatric services utilization by colleagues from Germany and other countries around the world, as well as experiences from the nonacute care settings, should be a lesson for mental health professionals, particularly those who believe that majority 
of patients with mental health problems are supposed to quickly decompensate in the time of global crisis. Mental illness does not necessarily affect resilience as a personality characteristic, particularly if there is a good balance of protective factors and risk factors. Finally, the internal experiences, sense of internal threat, and suffering from illness per se sometimes outweigh the level of perceived external threat and the experience of real danger. The last statement is a lesson from psychology and psychotherapy.

\section{Compliance with ethical standards}

Conflict of interest The author has no conflict of interest.

\section{References}

1. Godenberg MN, Parwani V (2020) Psychiatric emergency department volume during Covid-19 pandemic. Am J Emerg Med. https ://doi.org/10.1016/j.ajem.2020.05.088

2. Bojdani E, Rajagopalan A, Chen A, Gearin P, Olcott W, Shankar V, Cloutier A, Solomon H, Naqvi NZ, Batty N, Festin FD, Tahera D, Chang G, DeLisi LE (2020) COVID-19 pandemic: impact on psychiatric care in the United States. Psychiatry Res 289:113069

3. Olwill C, Mc Nally D, Douglas L (2020) Psychiatrist experience of remote consultations by telephone in an outpatient psychiatric department during the COVID-19 pandemic. Ir J Psychol Med $22: 1-8$ 